\title{
Existence of Quality and Testability of Quantity as Postulations in Educa- tional and Psychological Measurement System
}

\author{
Ying $\mathrm{Xu}^{*}$ and Huailong $\mathrm{Li}$
}

Department of Education, Huaibei Normal University, Huaibei, Anhui, 235000 China

\begin{abstract}
Any measurement system must be in statistical control and the confirmation of measurability is the basic theoretical hypothesis in educational and psychological measurement. We analyze some assumptions in three measurement models in psychological and educational system through the history analysis and comparative study. Based on this, we propose three postulations in educational and psychological measurement: psychological and educational measurement object is an objective existence in "quality"; the "quantity" relative stability of the measuring object is unity of "quantity" relative invariance in individual objects and "quantity" difference in group objects; hypothesis of measurability of quantity: Psychological and educational measurement object can be measured in the "quantity". The study improves the theoretical postulations and provides a solid foundation for educational and psychological system measurement.
\end{abstract}

Keywords: Psychological and educational measurement system, existence of quality, relative stability of quantity, testability.

\section{INTRODUCTION}

Any theoretical system is established on the basis of some basic assumptions. These assumptions constitute the axiom system (also known as postulation), taking it as a starting point, theory will get expansion and development, and then formed the theoretical system. Psychological and educational measurement theory is no exception; it is also based on some basic assumptions.

In twentieth century, psychological and educational measurement theory has developed the item response theory(ITR), generalizability theory(GT) and cognitive diagnosis model(CDM) on the basis of the classical test theory(CTT). The basic hypothesis of these theoretical models is to response the psychological and educational measurement theory premise, and is developing according to the practical need $[1,2]$. The basic hypothesis is different, but it has fundamental contributions for the development of the psychological and educational measurement theory, and has produced profound influence to the psychological and educational measurement practice. But these theoretical models can not replace the general theory of the psychological and educational measurement, or serve as the general hypothesis of the psychological and educational measurement.

In fact, in many classical literatures of psychological and educational measurement, it is regarded as psychological and educational measurement theory base for the measurement knowledge of the ancient Chinese ideologist Mencius (BC 327 - 289) and the western scholar E. L. Thorndike (1874-1949) and W. A. McCall(E.L. Thorndike, E.P.Hagan, 1985,p.2).
Mencius said, balance and then see the weight, measure and then know the length, everything is like this, especially psychology. That is to say, all phenomena, whether material or mental (psychological), there will be different degree, different degree can be only be put out by the measurement. In 1918, E. L. Thorndike put forward, "Whatever exists at all, exists is some amount" (Thorndike, 1918). In 1939, W.A. McCall further pointed out, "Anything that exists in amount can be measured" (McCall, 1939). Consider the two sentences, that is to say as long as there is quality, there is quantity. The existence of quantity can be measured. That is all the objective things can be measured.

The basic assumption of psychological and educational measurement theory is actually to answer "the question of the possibility of the measurement. It includes four aspects: (1) the existence of object cannot be doubted, namely the confirmation of the quality existence of the measured object; (2) the object to be measured is relatively stable in the "quantity"; (3) the "quantity" of different object is different, and change in a certain range; (4) testers (measuring people) can develop (design, develop, and constantly improve) the measuring tools and techniques for the measured object. However, the above understanding of measurement means that there are things, there are the amounts, and the amount can be measured. It failed to give a complete affirmative hypothesis of the "measuring the possibility ". It answered only partly the above 2 and 4 aspects. It is obviously not enough and rigorous to take these general understanding or even spoken express as the cornerstone of developed theory of psychological and educational measurement. Against the above four points, this paper tries to further clarify and definite the theoretical premise of psychological and educational measurement from epistemology view. 


\section{ASSUMPTIONS OF THREE CLASSIC MODELS IN PSYCHOLOGICAL AND EDUCATIONAL SYSTEM MEASUREMENT}

There are three classic models in psychological and educational measurement. Each has its own assumptions.

\subsection{Classical Test Theory}

Classical Test Theory which is based on True Score Theory has three assumptions. True score has immutability what means character being measured is stable or constant. Error is entirely stochastic so it can be offset when measurement times is enough. The observation data is true score plus error (as shown in formula 1). So true score equals to the score measured (as shown in formula 2) and true score and error is independent of each other(as shown in formula 3 ).

$$
\begin{aligned}
& X=T+E \\
& X_{(T)}=E(X) \quad \text { or } \quad E\left(X_{S E}\right)=0 \\
& \rho\left(X_{T}, X_{S E}\right)=0
\end{aligned}
$$

\subsection{Item Response Theory}

There are also three assumptions in item response theory proposed to make up the deficiencies in Classical Test Theory. They are unidimensionality in Latent Trait Space, local independence between items, and item characteristic curve. Item characteristic curve is the graph of item response function. The curve is especially important because item response theory is founded on some form of characteristic curve rather than theoretic function. Many facts proved that the characteristic curve is S-Curve that is determined by three parameters: guess parameter (c) difficulty parameter (b) and differentiation parameter (a) (as shown in Fig. 1).

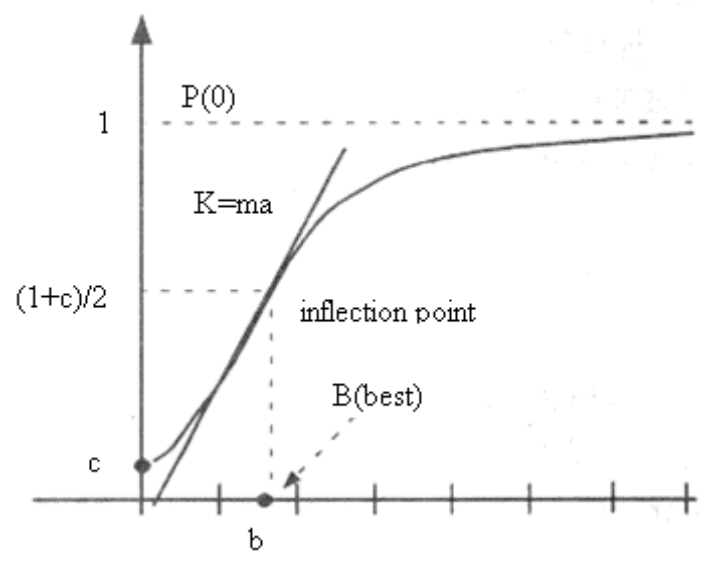

Fig. (1). Typical 3 ParametersCharacteristic Curve.

\subsection{Generalizability Theory}

Generalizability theory is raised in 1972(Cronbach, Gleser, Nanda, Rajaratnarn, 1972). It includes two main assumptions. On one hand, it uses dependability instead of reliability. Dependability means the character measured is stainable. On the other hand, observed number is change with other factor varying so we classify it into cross design, nest design and mixed design in practice (as shown in Figs. 2 and 3).

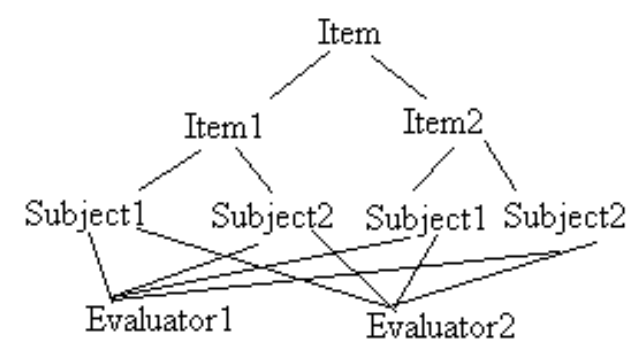

Fig. (2). Cross Design Model.

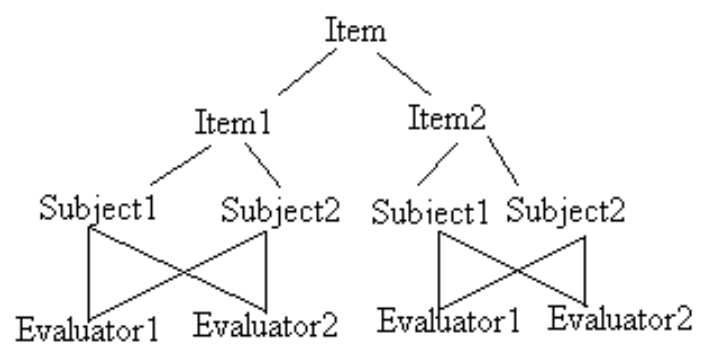

Fig. (3). Nest Design Model.

\section{THE "QUALITY" OBJECTIVE EXISTENCE OF THE MEASURED OBJECT}

From the epistemological perspective, anything is the unity of quality and quantity. Quality difference is the premise of the classification, and is the principle of things, however the differences in quantity is the premise of the measurement. If there is no difference there is no need to be measured. Here the things refers to not only the objective phenomenon outside, also refers to human inner subjective phenomena. [5] The object of psychological and educational measurement is mostly such subjective phenomena.

It is generally believed that psychological and educational measurement is "the quantitative description of the human psychology and educational achievement by test based on the theory of psychology and education [5]". So, the measured object is psychological traits or educational achievement, is the "quality" existence of the measured object. Only 'quality' exists that "quantity" can be described. Apparently, the objective existence of measured object "quality" is the basis for the implementation of measurement. If the existence of "quality" is in doubt, the basic premise of measurement ceased to exist. There is no measured object, there can be no amount description.

In fact, the "qualitative" existence of psychological and educational measurement object contains the following layers of meaning:

\subsection{Independent of Will or Understanding of People}

The "quality" existence of measured object refer to the "quality" of object to be measured exists not on the will or the understanding of people (measurement subject). The stipulation of quality of the objective thing is inherent of object, not fabricated out according to the human (or measurement subject) subjective volition (whether admit that or not, like that or not) or subjective knowledge. It doesn't change with subject cognize. Even if human beings expand and deepen knowledge of the quality of the object to be 
measured with the accumulation of experience and the progress of science and technology, and get further understanding, can not change the objective existence of the object to be measured.

\subsection{Independent of Testers}

The existence of "quality" measured object refers to the measured traits do not depend on the people (testers) measurement to exist. That is, the quality of the measured object is all there regardless of the implement of measurement. In educational and psychological measurement it is especially not easy to get a consensus. Because many psychological traits are often required to obtain their characteristics by measurement, it is easy to regard traits as something measured from the measuring tool (usually the operational definition of the measured objects). For example, in the research process of intelligence, the intelligence once was considered something measured by intelligence scale." The so-called intelligence is something from (intelligence) test (Edwin.Borings, 1923).

\subsection{Inner Subjective Phenomenon}

The "quality" existence of the measured object refers to not only the objective phenomenon outside, also the inner subjective phenomenon of the person. Although psychological and educational object of measurement is abstract, cannot be observed directly by the naked eye, or be invisible, intangible things, it is experienced by the human. It can be obviously perceived by the measured subject. For example, "fear" (is a human emotion experience) is existence that people experienced.

\subsection{Named or Logoed}

The existence of the measured object "quality" also refers to the "quality" of the measured object has been (measurement subject) named or logoed. The quality existence of the measured object also means it is already named by human according to the experience and understanding (also known as stick "tag"), usually be given the strict description or definition, the connotation and extension (between subjects) has reached consensus in a certain range, often is to do operational definition in measurement.

\subsection{Constructed Continuously}

The "quality" existence of measured object is completed by continuous construction of the measurement subject. Generally speaking, the quality existence of psychological and educational achievement is not always clear because of the abstractivity, subjectivity, uncertainty, and indirect way of measurement methods. The determination of objective existence is to be completed with the following two methods in psychological and educational measurement process.

Data-driven-approach: Researchers of psychological and educational measurement develop measurement tool based on the theory and explore the structure of the qualities of the measured by measuring tool, to obtain further profound understanding to the existence of the characteristics. In fact, this method is the mainstream in nearly a hundred years. This method is often called the "bottom-up" approach. Peo- ple are preparing intelligence test on basis of imperfect intelligence theory, preparing personality measurement on basis imperfect personality theory. In this case, the test measurement researchers compiled may rely too much on the acquisition data in the preparation process (Houcan Zhang, 1997, p.1-4).

Theory-driven-approach: The acquired theoretical conception of psychology and education experts through a lot of psychological and educational phenomena and processes is often the direct basis of judgment of objective existence. For example, intelligence structure model, the response characteristics etc. are the understanding on the objective existence of a given trait from philosophy idea, this method is also known as the "top-down" approach. With the development of cognitive psychology, psychologists pay more and more attention to the internal process and knowledge structure of human psychological activities, the study of intelligence, personality and each subject psychology has made new breakthroughs. And the test on psychological quality are also beginning to appear theory oriented methods trend, i.e. to complete the test preparation process on basis relatively mature psychology theory. And theoretical framework of measurement is considered as a part of the research on measurement theory (Houcan Zhang, 1997, p.1-4).

In fact, understanding(including the definition and identification) on the objective existence of "quality" of psychological traits is the result of an expanding and deepening process, to complete by changing the method of "top down" and "bottom up" and the stepwise iteration. The existence of "quality" is completed by continuous construction of the measured subject.

\section{TESTABILITY OF QUANTITY}

\subsection{The "Quantity" Relative Stability of The Measured Object}

Generally speaking, "measurement is description of quantity." [5]. Only the measured object is relatively stable, it can be measured.

The "quantity" relative stability of psychological and educational measurement object refers to the stable value of the individual measured object in a certain time and space conditions. If the "quantity" of the measured object is always changing, it can not be measured, or no significance of the measurement. The purpose of measurement is to know the scale or level of the objective things, and then understand the "quality" of objective things. If it has changed after measurement, there is no is necessary to measure, in fact it cannot be measured. That is to say, the "quantity" is stable for individual object, i.e. the "quantity" of the measured individual won't have too big wave under certain conditions, it remained relatively stable, even if there is some change, the change is negligible. Of course this does not mean that the "quantity" of tested individual does not change in different conditions. Therefore, the "quantity" is relatively stable and not absolute stability.

\subsection{Difference of "Quantity" of the Measured Object}

It is generally believed that "quantity difference of things is the premise of measurement"[5], that is, the quantity of the 
whole (overall or sample) being measured is different. If there is no difference, there is no need to be measured. Psychological characteristic and educational achievement are different as all other physical phenomena, the difference not only include the qualitative aspects, also includes quantity aspects. Because there are differences, there are the performances of a certain number, so it is necessary to measure the difference, describe the difference degree [5]. Differences in the "quantity" of different individual characteristics are the premise of measurement. If there is no difference, there is no need to measure.

The value of the "quantity" in the measured group characteristics changes in a certain range. In psychological and educational measurement process, in most cases, what the subject concerns is the distribution of "quantity" and data related characteristics of the measured group (e.g. mean value, standard deviation). The distribution and change" of quantity" of measured object group reflects certain regularity.

\section{DEVELOPMENT OF MEASUREMENT TOOLS}

Measurement is a kind of action mode of the measurement subject on the measurement object, it is the interaction between the subject and the object, and is the understanding of the subject to the object. On basis of knowability, the world can be recognized. Only by adhering to the knowability theory, one can consider the measuring subject will develop special measurement tools to measure the "quantity" of objective things. Also because of the particularity of psychological and educational measurement (subjectivity, abstract, variability of the object, indirect measurement method, etc.) it gives the psychological and educational measurement tools development the following special meaning:

\subsection{Tools Designed and Improved by Testers}

The development of measurement tools is that measurement subject can design, develop and improve the measurement tools. Against the specific object in educational and psychological measurement, measuring subject can design, develop, and perfecting the corresponding measurement tools and techniques according to the existing research in psychology and education theory, based on the full understanding of the measurement object, through specific methods and techniques of subjective efforts and tool development.

\subsection{Tools Measured Indirectly}

The development of measurement tools refers to that measurement tool is an indirect measurement tool for measuring object. Psychological and educational achievements are not physical objects, which cannot be measured directly. But they will be shown in the external behavior of the measured object(people), reflected by its external behavior. Therefore, people could measure differences in psychological traits and educational achievement through the external behavior observation. Under appropriate conditions, measurement subject can develop the development tools for measuring external behavior performance, and speculate the "quantity" of psychological traits and educational achievement through the measurement tools for external behavior.

\subsection{Tools Rrevised Continuously}

The development of measurement tools is a measurement tool in constant revision process. The accuracy, reliability and accuracy in psychological and educational measurement, as all other measurement technology, is relative. It is not a precise quantity without error, but a statistical estimate quantity of the measured object. The measurement subject may measure the quantity of the measured object. Although the error of the measurement is always theoretically there, the accuracy of the psychological and educational measurement will be getting higher with the progress of science and technology.

\subsection{Tools Dependent on Measuring Techniques}

Development of instrument tools depends on the measuring tools and techniques. Educational and psychological measurement maybe measure the "quantity" of the measured object by measurement tools and measurement technology, like other physical measurements. But psychological and educational measurement scientists need more effort to develop effective measuring tools and measurement technology. There are perfect tools for some phenomena, but still the measurement tools for some phenomena have not been invented. But we cannot say that some phenomena can not be measured for the tools have not been invented yet [4].

\section{CONCLUSION}

From the epistemological point of view, the theoretical premise of psychological and educational measurement should include the following three basic assumptions:

(1) Hypothesis of objective existence of quality: Psychological and educational measurement object is an objective existence in "quality".

(2) Hypothesis of relative stability of quantity: The "quantity" relative stability of the measuring object is unity of "quantity" relative invariance in individual objects and "quantity" difference in group objects.

(3) Hypothesis of measurability of quantity: Psychological and educational measurement object can be measured in the "quantity" (in the scale and level).

In a word, "for the object of psychological and educational measurement, the quality is objective existence; the volume is relatively stable and measurable". Only this is approved, psychological and educational measurement theory can be developed, psychological and educational measurement practice can be carried out.

\section{CONFLICT OF INTEREST}

The authors confirm that this article content has no conflict of interest.

\section{ACKNOWLEDGEMENTS}

This work is supported in part by "the Ministry of education of Humanities and Social Science project" Foundation 
of China under grant No.14YJC890026 and "the Anhui Philosophy and Social Science Planning Program" Foundation of China under grant No.AHSKQ2014D34.

\section{REFERENCES}

[1] Haiqi Dai, Feng Zhang, and Xuefeng Chen, Psychological and Educational Measurement, JiNan University Press, 2011.

[2] Zhongheng Dai, "Recent Development of Educational Measurement in China", Teacher Education research, vol.02, 1990, pp. 1520.

[3] R.L. Thorndike, E.P. Hagen, Measurement and evaluation in psychology and education, 4th edition. New York: John Wiley and Sons. 1977.

[4] Shulin Wang, Psychological and Educational Measurement, FuJian Education Press,2008.

[5] Guanghua Mo, What Is Intelligence, HuNan Science and Technology Press, 2001.

[6] Houcan Zhang, Jiayuan Yu, "The History of Psychological Testing Development in China", Psychological Science, 35(3), 2012, pp. 514-521.

[7] Onyon C. "Problem-based learning: a review of the educational and psychological theory", Clinical Teacher, 9(1) ,2012, pp. 22-26.

[8] SJ, Ersser, et al. "Psychological and educational interventions for atopic eczema in children.." h ohran Daaba Of yma Rvw 2014.

[9] Kane, Michael T, "Validating the Interpretations and Uses of Test Scores." Jornal of daonal Marmn 50.1,2013, pp.1-73.
[10] Brennan, Robert L," Commentary on "Validating the Interpretations and Uses of Test Scores"." Jornal of daonal Marmn 50.1,2013, pp.74-83.

[11] Hou, Likun, et al. "Differential Item Functioning Assessment in Cognitive Diagnostic Modeling: Application of the Wald Test to Investigate DIF in the DINA Model." Journal of Educational Measurement 51.1,2014, pp.98-125.

[12] Clare, Linda, et al. "The influence of psychological, social and contextual factors on the expression and measurement of awareness in early-stage dementia: testing a biopsychosocial model." International Journal of Geriatric Psychiatry 27.2,2012, pp.167-177.

[13] Gierl, Mark J, and Hollis Lai, "Instructional Topics in Educational Measurement (ITEMS) Module: Using Automated Processes to Generate Test Items." daonal Marmn: and ra 32.3,2013, pp.36-50.

[14] Hansen, Tina, et al, "Validation of the Danish version of the McGill Ingestive Skills Assessment using classical test theory and the Rasch model." Dably and Rhablaon 34,2012, pp.859-868.

[15] Kosinski, Mark, et al. "Applying Item Response Theory Methods to Improve the Measurement of Fatigue in a Clinical Trial of Rheumatoid Arthritis Patients Treated with Secukinumab." Open Journal of Rheumatology \& Autoimmune Diseases 4,2013, pp.192-201.

[16] Marshall, Ineke Haen, and Dirk Enzmann. The Many Faces of Youth Crime. Springer New York, pp.285-325,2012.

[17] Briesch, Amy M., et al. "Generalizability theory: A practical guide to study design, implementation, and interpretation." Jornal of hool yhology 1,2014, pp.13-35.

(C) Xu and Li; Licensee Bentham Open.

This is an open access article licensed under the terms of the Creative Commons Attribution Non-Commercial License (http://creativecommons.org/licenses/by-nc/3.0/) which permits unrestricted, non-commercial use, distribution and reproduction in any medium, provided the work is properly cited. 